\title{
OBSTETRIC HEMORRHAGE IS AS ONE OF THE CAUSES OF MATERNAL DEATHS
}

Irina Akperbekova1 ${ }^{*}$, Bekhzod Abdullaev#

${ }^{1}$ No.1 Department of Obstetrics \& Gynecology, Tashkent Medical Academy, Uzbekistan

${ }^{2}$ Scientific Researcher, $6^{\text {th }}$ Year Student GP Faculty, Tashkent Medical Academy, 2 Str. Farobi, Tashkent, Uzbekistan. 100109

Corresponding author author.uzb@mail.ru (I. Akperbekova), abdullayevbehzod@mail.ru (B. Abdullaev)

Abstract: Obstetric hemorrhage as a cause of maternal morbidity and mortality, over a long period it is one of the leading places in the world and among the five major causes of maternal mortality (MM). We aimed to analyze the reasons for $\mathrm{MM}$ in obstetric hemorrhage and to determine the complex of measures on prevention of maternal mortality from obstetric hemorrhage. Clinical,

\section{Health Sciences}


retrospective clinical and statistical analysis of data were studied in 67 stories of births ended in maternal mortality from obstetric hemorrhage. The dead women were aged 20-29 years- 38 (57\%), 30-35 - 19 (28\%), over $36-10(15 \%)$. By parity of predominated over repeatedly pregnant. The first pregnancy was $23(34.3 \%)$, repeatedly pregnancy - 25 $(37.3 \%)$ and multiple pregnant women - 19 (28.4\%). Causes of bleeding were placenta abruption - in 31 (46\%), placenta previa in 2(3\%), Hypo - or atonic bleeding in 24 (36\%), injuries of the birth canal - in 6(9\%), intra-abdominal vascular bleeding - 2 (3\%), coagulopathies bleeding - in 1 $(1.5 \%)$ women. Bleeding due to an ectopic pregnancy in 1 $(1.5 \%)$. Bleeding in the late postpartum period occurred in $5(8 \%)$ women. Bleeding is diagnosed late in 4 due to the fact that in 3 cases, the women were not observed after birth $(14 \%)$ and in 1 case $(1.5 \%)$ - after the surgery. The most frequent omissions and unrecorded features were late admission to hospital in the state of hemorrhagic shock in $40 \%$. Late deliveries were in $31 \%$; delayed cesarean section were in $27 \%$; bleeding after cesarean section was in $25 \%$; wrong choice of surgery volume in $22 \%$; underestimation of blood loss in 52\%; non-compliance with protocols in $54 \%$, non-surgical method for stopping bleeding was $12 \%$, not the source of bleeding was $6 \%$. These data indicate the need to improve emergency care in maternity institutions. We can deduce the following recommendations for the prevention of mortality from hemorrhage: improvement of antenatal care and the treatment of pregnant women of groups with risk factors for bleeding, in the first place, suffering extragenital diseases and training of obstetricians and gynecologists.

Keywords: Maternal Mortality, Obstetric 
Hemorrhage, Extragenital Diseases

\section{Introduction}

Obstetric hemorrhage as a cause of maternal morbidity and mortality, over a long period occupies one of leading places in the world and it is among the five major causes of maternal mortality. In despite of significant advances development and implementation of clinical protocols are the basis of life saving and recovery of patient in efficient and well-organized work multidisciplinary team capable of providing timely and quality medical care. An important role in this sense, the play worked out unified approaches and standards for the provision of skilled care. Huge efforts of the Ministry of Health, leading experts and international organizations have a goal to reduce the maternal mortality rate in Uzbekistan through the introduction of new technologies, methods of prevention and treatment of the main types of obstetric pathology [21].

Early postpartum hemorrhage accounts for a large part of all obstetric hemorrhages [5, 8]. According to WHO, annually 127 thousand women $(25 \%)$ in the world die from bleeding. According to some authors, obstetric hemorrhage as the primary cause of maternal mortality is in pure form $20-25 \%$, as a competing cause of $42 \%$, as a background to $78 \%[18,22]$.

\section{Material and Methods}

\subsection{Material}

A retrospective analysis of 67 confidential birth history, ending maternal mortality from obstetric hemorrhage in 2014-2015 in the Republic of Uzbekistan by

\section{Health Sciences}


random sampling using developed by use of the program.

\subsection{Patient Statistics}

The dead women were aged 20-29 years- 38 (57\%), $30-35$ - 19 (28\%), over $36-10(15 \%)$. The social status of Housewives was the most - 57 women (85\%), 10 employees $(15 \%)$. In the outpatient sector on the account consisted of 63 pregnant women, obstetrician-gynecologist was observed by 24 women (35.8\%), had a record $4(6 \%)$. Of those who consisted on the account, visited the doctor 4 times - $28(42 \%)$, and 5 or more times - 35 women (52\%).

For delivery came in the obstetric Department of level 1 - 24 (36\%) women, 2 level - 33 (49\%) and 3 level - 10 $(15 \%)$. The place of death occurred in the institution of the $1^{\text {st }}$ level in 21 cases $(31 \%), 2^{\text {nd }}$ level $-24(36 \%)$ and $3^{\text {rd }}-22$ $(33 \%)$.

By parity of predominated over repeatedly pregnant. The first pregnancy was $23(34.3 \%)$, repeatedly pregnancy $25(37.3 \%)$ and multiple pregnant women - $19(28.4 \%)$.

The gestational age was 22 weeks in $2(3 \%), 23-28$ weeks in 5 cases $(7.5 \%), 28-32$ weeks in $8(12 \%)$, and $33-37$ weeks 19 (28.2\%), 37 and more weeks in 33 cases (49.3\%) pregnant women. Thus, $1 / 2$ of the deceased women had a full-term pregnancy.

From the extragenital diseases in women has been revealed anemia in $49(73 \%)$ pregnant women: mild in 11 $(16 \%)$, average in $15(22 \%)$, and severe - in $23(34 \%)$. With chronic pyelonephritis was suffered $7(10 \%)$ women, the varicose disease is established in $2(3 \%)$, and 1 case revealed rheumatism, thyroid disease, cardiovascular system and bronchial asthma. Hypertensive status was detected in 29 (43\%) pregnant women with pre-eclampsia in $25(37 \%)$ and eclampsia in $3(5 \%)$.

\section{Health Sciences}




\section{Results}

Births occurred in 66 women, of which vaginal in 21 $(32 \%)$, elective caesarean section was made in $2(3 \%)$ and emergency caesarean section in 43 (65\%). 1 woman died from an ectopic pregnancy. Indications for cesarean section were: placenta abruption in $24(36 \%)$ cases. Severe preeclampsia in 7 cases (11\%), eclampsia in 2 cases $(3 \%)$, uterine scar - $4(6 \%)$, uterine rupture in $2(3 \%)$, inconclusive for the fetus found in $2(3 \%)$, pelvic-disproportion of head in $3(5 \%)$, placenta previa in $1(1,5 \%)$, liver in 1 case $(1,5 \%)$, unsatisfactory progress in labor - in $1(1,5 \%)$.

Causes of bleeding were placenta abruption - in 31 $(46 \%)$, placenta previa in $2(3 \%)$, Hypo - or atonic bleeding in $24(36 \%)$, injuries of the birth canal - in 6(9\%), intraabdominal vascular bleeding $-2(3 \%)$, coagulopathies bleeding -in $1(1.5 \%)$ women. Bleeding due to an ectopic pregnancy in $1(1.5 \%)$.

Bleeding in the late postpartum period occurred in 5 $(8 \%)$ women. Bleeding is diagnosed late in 4 due to the fact that in 3 cases, the women were not observed after birth $(14 \%)$ and in 1 case $(1.5 \%)$ - after the surgery.

The volume of blood loss up to 11 was $11 \%$ of the cases, from 1 to 1, 51 in 30\%, from 2 to 2.5 liters is $34 \%, 3$ liters or more is $19 \%$, and it was impossible to determine the amount of blood loss in $6 \%$ of cases.

The number of surgeries was as follows: ligation of three pairs of vessels was performed in $27(40 \%)$ cases, the seam along B-Lynch was applied in $6(9 \%)$, amputation of the uterus produced in 11 (16\%) women, hysterectomy 27 patients $(40 \%)$, and relaparotomy in $18(27 \%)$ and rerelaparotomy - in $5(8 \%)$ cases. Late surgical assistance was provided to $44(66 \%)$ women.

\section{Health Sciences}




\section{Discussion}

The low level of skilled medical care exemplified by the following: underestimation of blood loss was detected in $52 \%$ [12], the delay of the operation was in $58 \%$ of cases, the management guidelines in $61 \%$ of cases, Infusiontransfusion therapy was not adequate in $58 \%$, and blood transfusion was not made in time $43 \%$ of women. These data indicate the necessity of improvement of emergency care in maternity institutions.

These data indicate the need to improve emergency care in maternity institutions. According to the study of MM in France (2013)[9], a 10-year period of inadequate assistance of $80-90 \%$ was determined fatal in women with obstetric hemorrhage and hypertensive disorders. According to H. Lombaard et al. (2009), in the cases of MM from postpartum haemorrhage, inadequate medical assistance was more often registered.

The most frequent omissions and unrecorded features: late admission to the hospital in a state of hemorrhagic shock - $40 \%$, late deliveries - 31\%, delayed cesarean section $-27 \%$, bleeding after cesarean section $25 \%$, the incorrect choice of operation volume - $22 \%$, underestimation of blood loss is $52 \%$, the failure of the protocols is $54 \%$, not produced by surgical method to stop bleeding at $12 \%$, is not installed the source of bleeding was $6 \%$ [6].

Traditional measures to stop hemorrhage are immutable, but they must be supplemented by modern methods [23, 1]. The interdisciplinary questions of continuity is extremely important, because a successful outcome in the fight against obstetric bleeding is hampered by the lack of sequence of actions that requires the 
integrated work of obstetricians, anesthesiologists, surgeons, assistance, ranging from prevention and ending late transfer to the surgical stage, the rejection of organ preserving surgery [16]. With the ineffectiveness of conservative treatment is extremely important to choose the time for radical intervention [2, 4]. The scope of surgical intervention is a question that is constantly debated in the pages of domestic and foreign literature [14, $15,17,3]$. It should be noted that, despite the use of such methods stop atonic uterine bleeding, as a superposition of compression sutures, ligation of the vessels of the uterus, the frequency of hysterectomies in the world is not only not reduced, but on the contrary increases. So, in Israel hysterectomy were as follows: $0.04 \%$ of all births in 19881994 years, a $0.05 \%$ in 1995-2000 and 0, $095 \%$ in 2001-2007 [7]. In the USA the frequency of hysterectomies equal to $0.05 \%$ of the total number of births and $0.5 \%$ of the number of caesarean sections [10]. Population-based study in Washington state showed an increase in the frequency of hysterectomy with $0.25 \%$ in 1987 to $0.82 \%$ in 2006 $(p=0.001)$. The main indication for surgery hysterectomy bleeding associated with previa, delay parts of the placenta, atony, uterine rupture and other causes [11].

There were transferred to the Emergency Department 12 $(18 \%)$, in departments of an artificial kidney on hemodialysis were 4 (6\%) women.

The post-mortem (pathological anatomy) examination was made in only $19(28 \%)$ cases.

The possibility of preventing MS depends on several factors: how fast was provided to obstetric care, training of specialists, medical personnel, medicines, equipment [13, 19, 20, 24]. 


\section{Conclusion}

Recommendations for the prevention of bleeding from MM: improving antenatal care - treatment group of pregnant women with risk factors for bleeding in the first place, suffering with extragenital disease. The training of obstetricians-gynecologists on the issue of "Obstetric hemorrhage: diagnosis, management of pregnancy, childbirth and after delivery" and a clear knowledge of protocols; improvement of the operative technique of cesarean section and hysterectomy; adequate assessment of blood loss and indemnity in accordance with existing protocols; the presence in each maternity facility of a stock of blood components, colloids and crystalloids; increasing medical knowledge, strengthening of explanatory work among the population by threatening conditions in pregnant women with mass media, National governmental Organisations and communities.

\section{References}

[1] Advances in the treatment of postpartum hemorrhage: review / A. M. El Ayadi, N. Robinson, S. Geller, S. Miller / / Expert Rev. Obstet. Gyn. - 2013. - Vol. 8, № 6. - P. 525-537.

[2] Bakri balloon effectiveness for postpartum hemorrhage: a "real world experience" / R. Olsen, D. P. Reisner, T. J. Benedetti, R. F. Dunsmoor-Su // J. Matern.-Fet. Neonat. Med. - 2013. - Vol. 26, № 17. - P. 1720-1723.

[3] B-Lynch suture in the management of massive post partum hemorrhage / S. Sheikh, S. Naz, A. Shaikh et al. // Rawal Med. J. - 2013. - Vol. 38, № 4. - P. 404-408. 
[4] Dabelea, V. Intrauterine ballontamponade in the management of postpartum hemorrhage / V. Dabelea, P. M. Schultze, R. S. McDuffie // Amer. J. Perinat. 2007. - Vol. 24. - P. 359-364.

[5] Geller S. E., Adams M. G., Kelly P. J., Kodkany B. S., Derman R. J. Postpartum hemorrhage in resource-poor settings.// Int J Gynaecol Obstet 2006.- 92: 202.-11.

[6] Lombaard, H. Common errors and remedies in managing postpartum haemorrhage / H. Lombaard, R. Pattinson / / Best Pract. Res. Clin. Obstet. Gynaecol. 2009. - Vol. 23. - P. 317-326.

[7] Peripartum cesarean hysterectomy / A. Orbach, A. Levy, A. Wiznitzer et al. // J. Matern. Fetal Neonatal Med. - 2011. - Vol. 24. - P. 480-488.

[8] Shane Higgins. Obstetric haemorrhage Emergency Medicine 2003.- 15.- p. 227-231.

[9] Ten years of confidential inquiries into maternal deaths in France, 1998-2007 / M. Saucedo, C. Deneux-Tharaux, M.-H. Bouvier-Colle et al. // Obstet. Gynecol. - 2015. Vol. 122, № 4. - P. 752-760.

[10] The frequency and complication rates of hysterectomy accompanying cesarean delivery / C. S. Shellhaas, S. Gilbert, M. B. Landon et al. // Obstet. Gynecol. - 2009. - Vol. 114, № 2 (pt. 2). - P. 224-229.

[11] Factors associated with Peripartum hysterectomy / C. Bodelon, A. Bernabe-Ortiz, M. A. Schiff, S. D. Reed // Obstet. Gynecol. - 2009. - Vol. 114, № 1. - P. 115-123.

[12] Aylamazyan E. K. Another approach to the problem of obstetric hemorrhage / E. K. Aylamazyan, M. A. 
Repina, T. W. Kuzminikh // Journ. obstetrics and female diseases. - 2008. - Vol. 57, No. 3. - p. 3-11.

[13] Analysis of causes of maternal death : a guide for physicians / ed. by A. P. Milovanova. - M., 2012. p.228.

[14] Barkagan Z. S. Diagnosis and controlled therapy of hemostatic disorders / Z. S. Barkagan, A. P. Momot. M.: Newdiamed-AO, 2008. - p.292.

[15] Baryshev B. A. Infusion-transfusion therapy for postpartum haemorrhage : a guide for physicians / A. B. Baryshev, E. K. Aylamazyan. - SPb., 2008. - p55.

[16] Zlatovratskaya T. V. Reserves to reduce maternal and perinatal morbidity and mortality in the maternity ward of the General hospital : dis. ... doc. med. sciences : 14.00.01 / T. V. Zlatovratskaya. - M., 2008. - p 250.

[17] The complex of measures to reduce preventable maternal mortality : guidelines / Ministry of healthcare of the Russian Federation. - M., 2007. - 32 p.

[18] Lebedenko E. Y. Reserves to reduce maternal mortality at the present stage: abstract. dis. MD..- Rostov-onDon.-2010.-p44.

[19] Malkova O. G. Analysis of major errors in intensive care patients with severe obstetric pathology in the NICU RCH №1 - a retrospective analysis over 5 years / O. G. Malkova, A. L. Levit // Intensive. therapy. 2012. - No. 3. - p 163-169.

[20] Experience of treatment of patients with obstetric pathology, complicated by multiple organ failure / E. F. Cherkasskaya, O. G. Malkova, V. V. Kovalev and others 
// Materials of the IV Congress of obstetricians and gynecologists of Russia (Moscow, 30 Sept. - 2 Oct. 2014). - M., 2008. - p 279-280.

[21] The order of MOH of RUz. Dated 27.09.2014, № 428 "About the introduction of a confidential inquiry of critical cases (CICC), threatening the lives of women in obstetric institutions of the Ministry of health of the Republic of Uzbekistan".

[22] Repina M. A. Maternal mortality associated with obstetric hemorrhage and the problem of uterine hemostasis: the scientific edition.// Journal of obstetrics and female diseases.-Saint-Petersburg.-2015.№3.-p. 18-23.

[23] Serov V. N. Ways to reduce obstetric pathology / V. N. Serov // Obstetrics and gynecology. - 2010. - No. 5. p. 8-12.

[24] Sukhanova L. P. Clinacal - statistical analysis modern trends of maternal mortality in Russian/ L. P. Sukhanova, A. N. Yusupova, V. A. Glushenkova// Healthcare of Russian Federation. - 2013. - № 4. - p 1419.

\section{Health Sciences}

